\title{
A novel and generalized approach in the inversion of geoelectrical resistivity data using Artificial Neural Networks (ANN)
}

\author{
A Stanley Raj ${ }^{1, *}$, Y Srinivas ${ }^{2}$, D Hudson Oliver ${ }^{2}$ and D Muthuraj ${ }^{3}$ \\ ${ }^{1}$ Vel Tech Dr. RR \& Dr. SR Technical University, Avadi, Chennai 600 062, India. \\ ${ }^{2}$ Centre for GeoTechnology, Manonmaniam Sundaranar University, Tirunelveli 627 012, India. \\ ${ }^{3}$ M.D.T. Hindu College, Tirunelveli, Tamil Nadu, India. \\ ${ }^{*}$ Corresponding author.e-mail: stanleyraj_84@yahoo.co.in
}

The non-linear apparent resistivity problem in the subsurface study of the earth takes into account the model parameters in terms of resistivity and thickness of individual subsurface layers using the trained synthetic data by means of Artificial Neural Networks (ANN). Here we used a single layer feed-forward neural network with fast back propagation learning algorithm. So on proper training of back propagation networks it tends to give the resistivity and thickness of the subsurface layer model of the field resistivity data with reference to the synthetic data trained in the appropriate network. During training, the weights and biases of the network are iteratively adjusted to make network performance function level more efficient. On adequate training, errors are minimized and the best result is obtained using the artificial neural networks. The network is trained with more number of VES data and this trained network is demonstrated by the field data. The accuracy of inversion depends upon the number of data trained. In this novel and specially designed algorithm, the interpretation of the vertical electrical sounding has been done successfully with the more accurate layer model.

\section{Introduction}

The study of geoelectrical parameters is useful for identifying the model parameters of the subsurface earth, viz., true resistivity and thickness. The geoelectrical resistivity inversion problem requires an appropriate relationship between mathematical formulations and the real world model parameters. Without initializing the estimation of the model parameters of subsurface layer, the flexibility to accommodate the particular estimation is difficult. So a powerful tool is necessary to estimate the parameters. Several researches were trying to make the solution unique (Backus and Gilbert 1969; Satyendra et al. 1994). The nonlinear inversion problem always requires a fairly accurate solution that reflects real world phenomena.

Artificial neural network (ANN) can play a major role in digging out the mystery of earth science by means of previously learned examples. These networks have self-learning capability and are fault-tolerant as well as noise-immune and have applications in various fields (Sreekanth et al. 2009; Singh et al. 2010; Maiti et al. 2011). Some of the researchers experimented with artificial neural network tool in geophysical problems very recently (El Qady et al. 2001; Jimmy Stephen et al. 2004; Singh et al. 2005; Maiti and Tiwari 2008, 2009; Srinivas et al. 2010, 2012). However the ID resistivity inversion problem is successful

Keywords. Artificial neural network; resistivity inversion; feed-forward back propagation algorithm; layer model. 
only when the problem is subjected to a particular case study. Training datasets of ANN have always played a role in successful testing. In the case study-related problems, usually $70 \%$ of the dataset is taken for training and tested with $30 \%$ of original field data. But if the field taken for study is new and if we require large datasets, then this generalized approach of using ANN application tool can be used. This research paper projects a novel and generalized approach for any case studyrelated problem and overwhelms the confined field area application.

A very important feature of these networks is their adaptive nature, whose 'learning by example' replaces 'programming' in solving problems. This feature makes such computational models very appealing in application domains where one has little or incomplete understanding of the problem to be solved but where training data is readily available. ANNs are now being increasingly recognized in the area of classification and prediction, where regression model and other related statistical techniques have traditionally been employed. Here electrical resistivity data has been taken to interpret the layer model of the subsurface of the earth using ANN.

\section{Data acquisition using Schlumberger vertical electrical sounding method}

Direct current resistivity methods of geophysical exploration are in extensive use globally for aquifer mapping and estimation of aquifer parameters, viz., resistivity and thickness (Kosinky and Kelly 1981; Sri Niwas and Singhal 1981; Mazac et al. 1985; Yadav and Abolfazli 1998; Louis et al. 2002; Batte et al. 2008; Singh et al. 2010; Maiti et al. 2011; Srinivas et al. 2012). The physical basis of the resistivity method is based on the relative distribution of impressed current in the earth, controlled by subsurface resistivity distribution. The interpretation of vertical electrical sounding (VES) data remained confined to curve matching procedure through theoretical curves prepared using different computational methods (Flathe 1955; Van Dam 1964; Mooney et al. 1966; Ghosh 1971).

The DC resistivity technique measures the Earth's electrical resistivity by injecting an electric current into the ground. The electrodes (A and B) were usually metal stakes that were hammered into the ground. Two additional electrodes $(\mathrm{M}$ and $\mathrm{N}$ ) were used to measure the voltage generated by the injected electric current (figure 1). We can calculate the apparent resistivity, which represents the average resistivity of a layered Earth over a hemispherical volume located between the electrodes and with a depth approximately equal to the current electrode spacing. The Schlumberger array is a standard electrode configuration where the current electrodes are moved out some predetermined distance for every measurement, while the potential electrodes remain in place. In practice, at some point the current electrodes are at too great a distance and it becomes difficult to measure the potentials; so the potential electrodes are moved outwards as well.

In general, the characteristic sounding curves illustrated in figure 2 represent the multiple layers. On this assumption each of the four sets has particular properties that may be roughly classified. Types $\mathrm{H}$ and $\mathrm{K}$ have a definite minimum and maximum, indicating a bed, or beds, of anomalously low or high resistivity, respectively, at intermediate depth. Types A and Q show fairly uniform change in resistivity - the first, increasing and the second, decreasing with depth. Obviously, these curves may also be combined. It is generally possible to tell from the shape of the adjacent parts of the profile, which layer correspond to the maximum or minimum on the first two curve types. For $\mathrm{H}$ and K-type curves $\rho 1>\rho 2<\rho 3$ and $\rho 1<\rho 2>\rho 3$, respectively, and we may be able to draw some conclusions about relative values of $\rho 1$ and $\rho 3$ if the spread has been extended sufficiently. The A and Q-type curves correspond to $\rho 1<\rho 2<\rho 3$ and $\rho 1>\rho 2>\rho 3$, respectively. Some idea of the relative bed thicknesses may be obtained from the horizontal extent of the maxima and minima as well as the flanking portions in all cases. The coordinates of the extreme points in curves of types $\mathrm{H}$ and $\mathrm{K}$ (i.e., maximum or minimum $\rho_{a}$ and electrode separation) may be used with certain characteristic curves for three layers employing a particular electrode spread (Telford et al. 1990).

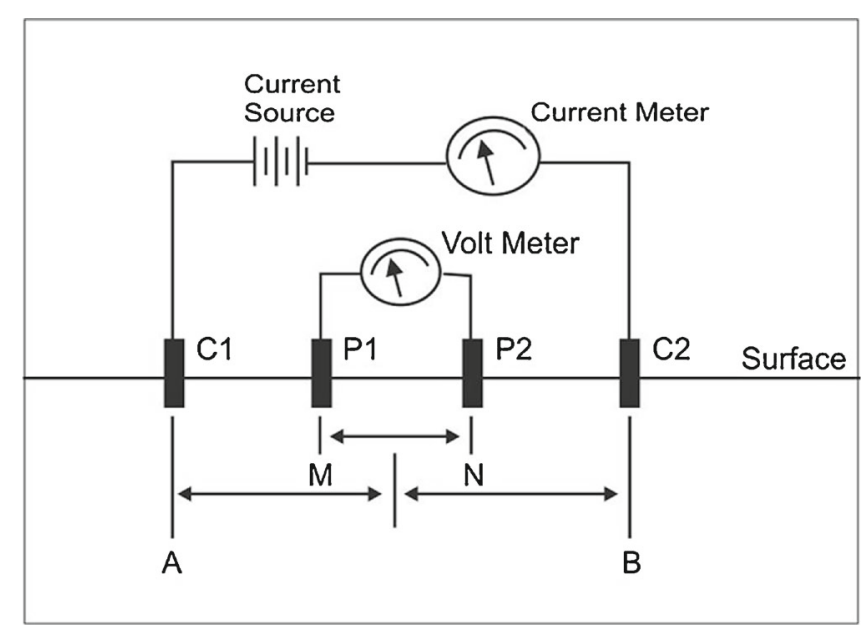

Figure 1. Schlumberger electrode configuration. 

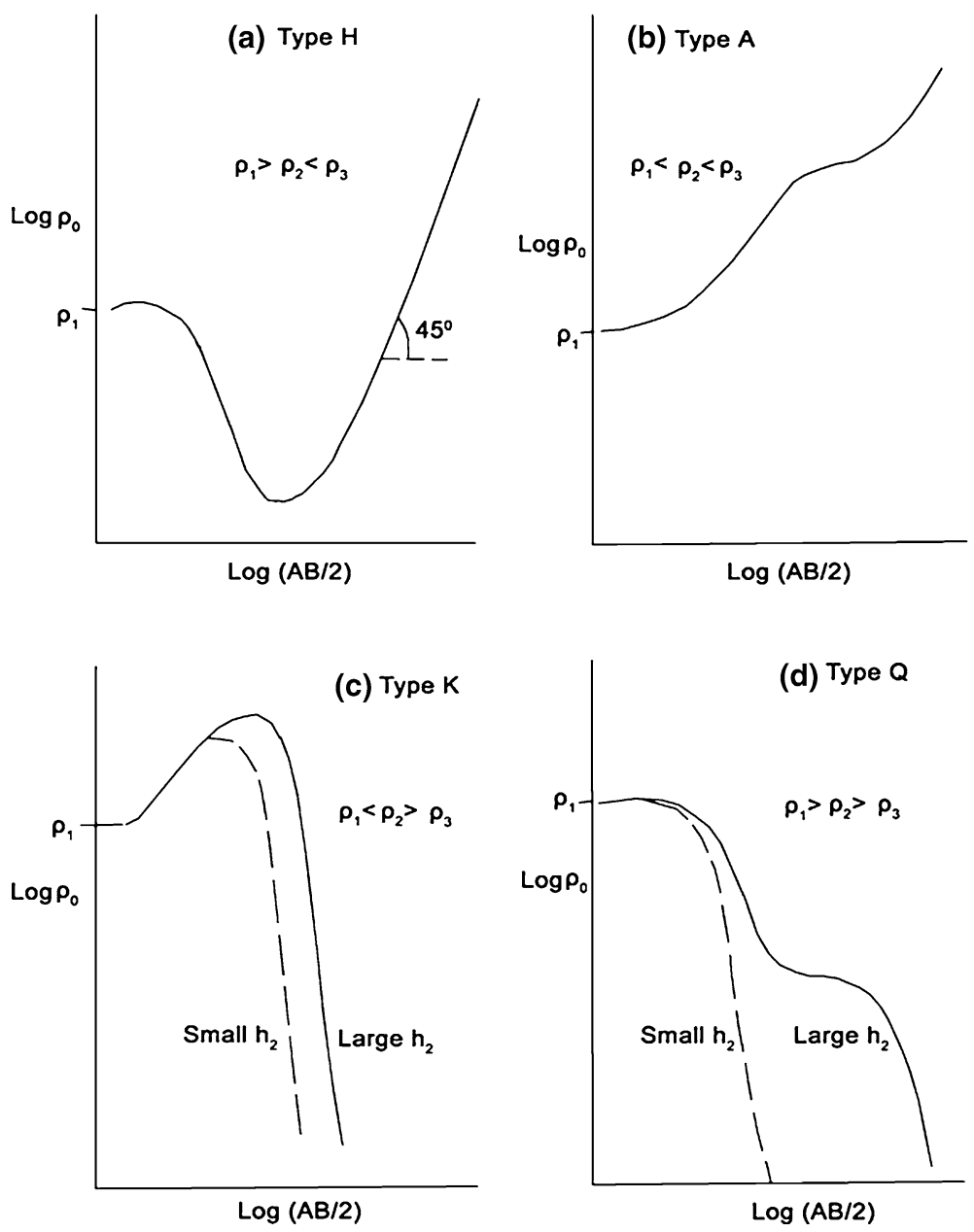

Figure 2. Characteristic sounding curve representing the four types of subsurface layers.

\section{Artificial Neural Network application on electrical resistivity method}

Interpretation of the electrical resistivity data is necessary to understand the concept of reality behind the earth subsurface system and there should be an efficient tool to estimate and predict the parameters which are closely relevant to the system. Thus, computational method involving the intelligent network to justify all the nonlinear problem is necessary. Conventional mathematical analysis is difficult when there are uncertain and partially defined systems with a degree of vagueness. The use of intelligent systems to evaluate such a complex system is ideal. An adaptive network consists of a structure of nodes and the directional links through which these nodes are connected. The outputs of the nodes depend on the parameters associated with these nodes. The change in these parameters is brought about by a learning rule to minimize the error. The learning techniques can automate this process and substantially reduce development time and cost while improving performance. For neural networks, the knowledge is automatically acquired by the back propagation algorithm and will be a more efficient tool used for the interpretation of geophysical data extensively.

Artificial neural networks have been successfully applied to a number of geophysical problems in this way, including parameter estimation, classification, filtering and optimization (Poulton et al. 1992; Sheen 1997; Vander Baan and Jutten 2000; Calderon-Macias et al. 2000; El Qady and Ushijima 2001). The adoption of the neural network approach holds several advantages over more conventional modelling techniques. It allows the effective solution of nonlinear problems from complex, incomplete and noisy data.

\section{Earlier research}

For 1D inversion of geoelectrical data batch back propagation algorithm was chosen and the resultant works of all back propagation paradigms were compared (El Qady et al. 2001). But further research on iterative feed forward network with three training algorithms (Singh et al. 2005) 
proved that the Levenberg-Marquardt algorithm is the best optimization algorithm for that particular case study problem. All the back propagation algorithm differs from each other in calculating the weights and updating (Werbos 1974).

Earlier research on geoelectrical inversion using ANNs is mainly for case study-related problems, because in applying ANN, one should have enough number of training datasets. Therefore forward modelling approach has been used extensively in earlier days. To have a good starting model, various techniques have been incorporated with the ANN architecture (Maiti and Tiwari 2008, 2009; Maiti et al. 2012, 2013). Here geoelectrical direct inversion has been attempted by means of ANNs. To have a large number of training data, ANN's specially designed algorithm has the power to generate synthetic data of its own. Apart from using the forward modelling techniques, ANN self generated algorithm thus provides a generalized approach to solve any geoelectrical field dataset and the same has been validated successfully in this paper.

Many training algorithms were tested with the help of powerful scientific software MATLAB and the following are the verified algorithms listed in the published research work of Srinivas et al. 2012: gradient descent back propagation (traingd), gradient descent with momentum back propagation (traingdm), one step secant back propagation (trainoss), BFGS-quasi newton back propagation (trainbfg) (BFGS refers to updating Broyden, Fletcher, Goldfarb and Shanno algorithm), training with scaled conjugate gradient back propagation (trainscg), training with conjugate gradient back propagation with Powell-Baale restarts (traincgb) and with Polak-Ribiere updates (traincgp) and with Fletcher-Reeves updates (traincgf), resilient back propagation (trainrp), training gradient descent back propagation with momentum and adaptive learning rate with back propagation (traingdx), training gradient descent with adaptive learning rate back propagation (traingda). The terms used in brackets are the commands used in the programming language of MATLAB software.

Robust behaviour of the feed forward back propagation with Levenberg-Marquardt training algorithm was comparatively proved to be the best performance algorithm (Srinivas et al 2012).

\section{Feed-forward back propagation with Levenberg-Marquardt (FFBP-LM) training}

Back propagation learning algorithm works for feed-forward networks with continuous output. Training starts by setting all the weights in the network to small random numbers. Now, for each input example the network gives an output, which starts randomly. We measure the squared difference between this output and the desired output. The sum of all these numbers of the output over all training examples is called the total error of the network. By choosing the weights that minimize the total error, one can obtain the neural network that best solves the problem at hand.

The advent of back propagation algorithm (BP), the adaptation of the steepest descent method, opened up new avenues of application of multilayered ANN for many problems of practical interest. A multilayer ANN contains three basic types of layers: input layer, hidden layer(s) and output layer. Basically, the back propagation learning involves propagation of error backwards from the output layers to the hidden layers in order to determine the update for the weights leading to the units in the hidden layer(s).

The nonlinear relationship between input and output parameters in any network requires a function, which can appropriately connect and/or relate to the corresponding parameters. In operation, each unit of an ANN receives inputs from other connected units and/or from an external source. A weighted sum of the inputs is computed at a given instant of time. The activation value determines the actual output from the output function unit, i.e., the output state of the unit. Input element vectors with a layer of neurons are shown in figure 3 . The output values and the other external inputs in turn determine the activation and output states of the other units.

The feed forward neural network was the first and arguably simplest type of artificial neural network devised. In this network, the information moves in only one direction, forward, from the input nodes, through the hidden nodes (if any) and to the output nodes. There are no cycles or loops in the network.

Back propagation learning involves propagation of the error backwards from the output layer to the hidden layers in order to determine the update for the weights leading to the units in a hidden layer. The error at the output layer itself is computed using the difference between the desired output and the actual output at each of the output units. The actual output for a given input training pattern is determined by computing the outputs of units for each hidden layer in the forward pass of the input data (Yegnanarayana 2005). A trained multiplayer feed forward neural network is expected to capture the functional relationship between the input-output pattern pairs in the given training data. It is implicitly assumed that the mapping function corresponding to the data is a 
smooth one. But due to limited number of training samples, the problem becomes an ill-posed problem, in the sense that there will be many solutions satisfying the given data, but none of them may be the desired/correct one (Tikhonov and Arsenin 1977; Weiland and Leighton 1987). Figure 4 illustrates the basic idea of fixing the function during training. Given the samples marked ' $x$ ', the objective is to capture the function represented by the solid curve. But depending on the size of the network, several solutions are possible, including the overtraining situations (shown by dotted curve) in which for all the training data the error is zero. In fact there could be several functions passing through the given set of points, none of which is the desired one. This happens if the number of free parameters (weights) of the network is very large. Such a situation results in a large error when some other (test) samples are given to validate the network model for the function. This is called 'poor generalization' by the network. On the other hand, less number of the free parameters may result in a large error even in the training data, and hence a poor approximation to the desired function. The function approximation interpretation of a multiplayer feed forward neural network enables us to view different hidden layers of the network performing different functions.

In this approach, ANN determines the exact curve while training the VES data. Permissible error percent used in the algorithm controls the overfitting situations during training of apparent resistivity data. If the fitting is poor, the error will be more and the entire algorithm will iterate again to manage the poor generalization of the network functions. More the number of epochs, less the poor generalization of the network. Apparent resistivity curve collected from the field contains field errors/noises due to the heterogeneity of the subsurface earth. This can be tackled by ANN inversion in terms of early stopping of the networks when the performance goal is reached. And also the learning rate and momentum thus controls the network to travel in the path of converging the results. Thus the curve with maximum fitting will be obtained as an output in the specially designed ANN geoelectrical inversion algorithm.

It is very essential for the networks to have a good selection of training parameters (Baum and Hausler 1989). In FFBP algorithms, the learning rate is a small number (Aristodemou et al. 2005) that controls the amount of error, since the interconnection of weights are negatively added to the network for the next iteration (Cranganu 2007). If the learning rate is large, then large changes are allowed in the weight changes and learning does not take place. On the other hand, if the learning rate is small, only small changes are allowed, which can increase the learning time and the network can run at the maximum number of epochs that the user fixes. But before the goal is achieved, the network stops training and brings out the best performance output. The momentum, $\alpha$, is a term that dampens the amount of weight change by adding in a portion of the weight change from the previous iteration (Singh et al. 2005). The main purpose of the momentum is to accelerate the convergence of the error propagation algorithm. It is used to prevent the system from converging to a local minimum. The momentum and learning rate terms were analyzed by Ahmad Neyamadpour et al. (2010) and suggested the learning rate of 0.01 , and a momentum coefficient of 0.2 .

The momentum coefficient with low value cannot reliably avoid local minima, but slows down the

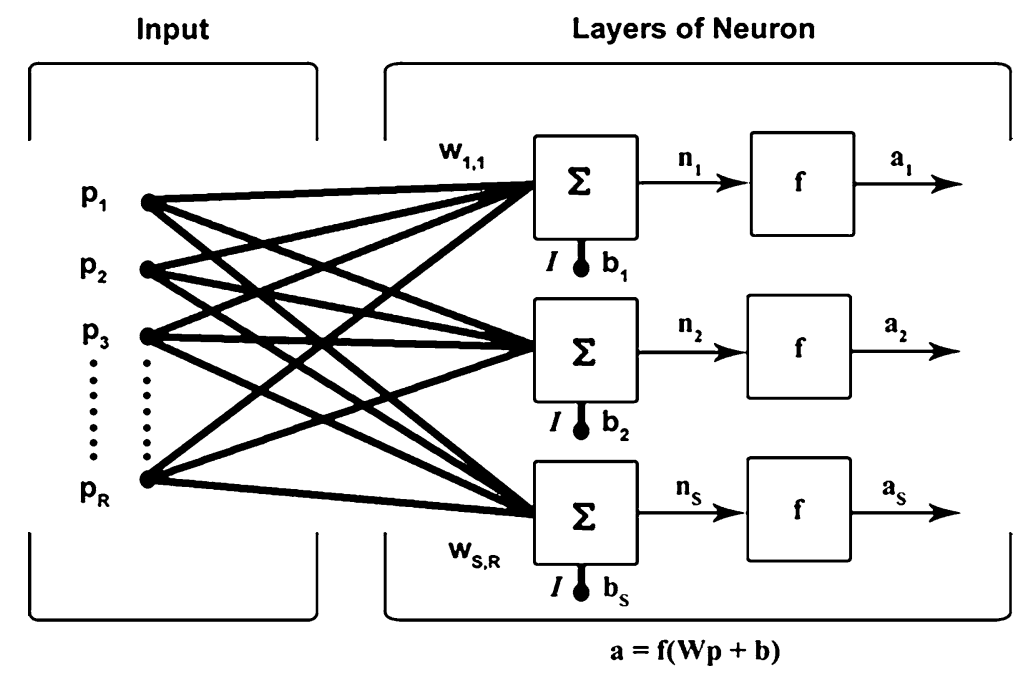

Figure 3. Input element vectors with layer of neurons having certain weights and bias. 


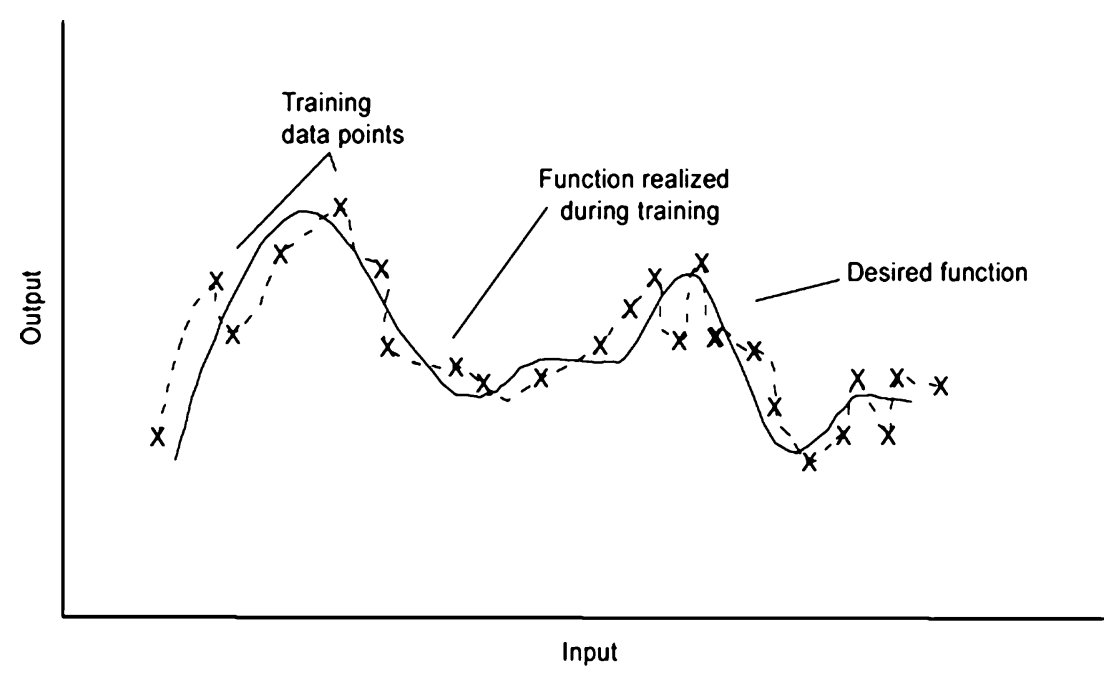

Figure 4. Neural network realized the function during training and fixes the appropriate function for the respective data.

training of the system. Though a high momentum parameter can help to increase the speed of convergence of the system, if too high it can create a risk of overshooting the minimum, which can cause the system to become unstable. So it has been maintained as 0.5 in this problem to stabilize the system for interpreting the geoelectrical resistivity data.

\subsection{FFBP-LM - theoretical background}

Feed-forward back propagation (BP) algorithm training involves two phases (Werbos 1974; Rumelhart et al. 1986).

- Forward phase: During this phase, the free parameters of the network were fixed, and the input signal was propagated through the network. The forward phase was finished with the computation of an error signal

$$
e_{i}=d_{i}-y_{i}
$$

where $d_{i}$ is the desired response and $y_{i}$ is the actual output produced by the network in response to the input $x_{i}$.

- Backward phase: During the second phase, the error signal $e_{i}$ was propagated through the network in the backward direction, hence the name of the algorithm. It is during this phase that adjustments were applied to the free parameters of the network so as to minimize the error $e_{i}$ in a statistical sense. The back propagation learning algorithm was simple to implement and computationally efficient in that its complexity was linear in the synaptic weights of the network. However, a major limitation of the algorithm was that it does not always converge and can be excruciatingly slow, particularly when we have to deal with a difficult learning task that required the use of a large network.

Feed-forward back propagation (FFBP) algorithm is the technique used here for studying the field data by means of a training function which updates weights with the specified learning function. Thus the network is trained with synthetic electrical resistivity data in the present study. Training large number of data will provide us good results of the problem and make the hidden units train the data more effectively (Satish Kumar 2007).

\subsection{Training algorithm}

The training algorithm of back propagation involves four stages:

1. Initialization of weights

2. Feed forward

3. Back propagation of errors

4. Updation of the weights and biases

Figure 5 shows the specially designed ANN generalized algorithm used for 1d geoelectrical resistivity inversion. During first stage, which is the initialization of weights, some small random values are assigned. During feed forward stage each input unit $\left(x_{i}\right)$ receives an input signal and transmits this signal to each of the hidden units $z_{1} \ldots z_{p}$. Each hidden unit then calculates the activation function and sends its signal $z_{j}$ to each input pattern.

During the back propagation of errors, each output unit compares its computed activation $y_{k}$ with its target value $t_{k}$ to determine the associated error for that pattern with that unit. The target is the best fitted apparent resistivity data on comparing 
with the original field data. Based on the error, the factor $\delta_{k}(k=1, \ldots, m)$ is computed and is used to distribute the error at output unit $y_{k}$ back to all units in the previous layer. Similarly, the factor $\delta_{j}(j=1, \ldots, p)$ is computed for each hidden unit $z$.

During the final stage, the weight and biases are updated using the $\delta$ factor and the activation.

The various parameters used in the training algorithm are as follows:

$x$ : Input training vector (in primary phase (figure 5) $\mathrm{AB} / 2$ as input and in major class training phase $\mathrm{AB} / 2$ and $\rho_{a}$ of synthetic data has been taken)

$$
x=\left(x_{1}, \ldots, x_{i}, \ldots, x_{n}\right)
$$

$t$ : Output target vector (in primary phase (figure 5) $\rho_{a}$ as output and in major class training phase true resistivity and depth of synthetic data has been taken).

$$
\begin{gathered}
t=\left(t_{1}, \ldots, t_{k}, \ldots, t_{m}\right) \\
\delta_{k}=\text { error at output unit } y_{k} \\
\delta_{j}=\text { error at hidden unit } z_{j} \\
\alpha=\text { learning rate } \\
V_{o j}=\text { bias on hidden unit } j \\
z_{j}=\text { hidden unit } j \\
w_{o k}=\text { bias on output unit } k \\
y_{k}=\text { output unit } k .
\end{gathered}
$$

The training algorithm used in the back propagation network is as follows. The algorithm is given with various phases.

\subsection{Initialisation of weights}

Step 1: Initialize weight to small random values. Step 2: When stopping condition is false, do Steps $3-10$.

Step 3: For each training pair do Steps 4-9.

Feed Forward

Step 4: Each input unit receives the input signal $x_{i}$ and transmits this signal to all units in the layer above, i.e., hidden units. Here in this approach, hyperbolic tangent sigmoid transfer function ('tansig' command used in the MATLAB software) activation function is used between the input and hidden layers of ANN algorithm. For the hidden to the output layer, linear transfer function is used ('purelin' command as used in the MATLAB software).
Step 5: Each hidden unit $\left(z_{j}, j=1, \ldots, p\right)$ sums its weighted input signals

$$
z_{-i n j}=v_{o j}+\sum_{i+1}^{n} x_{i} v_{i j} .
$$

Applying an activation function

$$
z_{j}=f\left(z_{i n j}\right)
$$

and sends this signal to all units in the layer above, i.e., output units.

Step 6: Each output unit $\left(y_{k}, k=1, \ldots, m\right)$ sums its weighted input signals,

$$
y_{-i n k}=w_{o k}+\sum_{j=1}^{p} z_{j} w_{j k}
$$

and applies its activation function to calculate the output signals.

$$
y_{k}=f\left(y_{-i n k}\right) .
$$

\section{Back propagation of errors}

Step 7: Each output unit $\left(y_{k}, k=1, \ldots, m\right)$ receives a target pattern corresponding to an input pattern, the error information term is calculated as:

$$
\delta_{k}=\left(t_{k}-y_{k}\right) f\left(y_{-i n k}\right) .
$$

Step 8: Each hidden unit $\left(z_{j}, j=1, \ldots, n\right)$ sums its delta from units in the layer above

$$
\delta_{-i n j}=\sum_{k=1}^{m} \delta_{j} w_{j k} .
$$

The error information term is calculated as:

$$
\delta_{j}=\delta_{-i n j} f\left(z_{-i n j}\right) .
$$

\subsection{Updation of weight and biases}

Step 9: Each output unit $\left(y_{k}, k=1, \ldots, m\right)$ updates its bias and weights $(j=0, \ldots, p)$.

The weight correction term is given by

$$
\Delta W_{j k}=\alpha \delta_{k} z_{j}
$$

and the bias correction term is given by

$$
\Delta W_{o k}=\alpha \delta_{k} .
$$

Therefore,

$$
\begin{aligned}
& W_{j k}(\text { new })=W_{j k}(\text { old })+\Delta W_{j k}, \\
& W_{o k}(\text { new })=W_{o k}(\text { old })+\Delta W_{o k} .
\end{aligned}
$$

Each hidden unit $\left(z_{j}, j=1, \ldots, p\right)$ updates its bias and weights $(I=0, \ldots, n)$. 
The weight correction term:

$$
\Delta V_{i j}=\alpha \delta_{j} x_{i} .
$$

The bias correction term:

$$
\Delta V_{o j}=\alpha \delta_{j} .
$$

Therefore,

$$
\begin{aligned}
V_{i j}(\text { new }) & =V_{i j}(\text { old })+\Delta V_{i j}, \\
V_{o j}(\text { new }) & =V_{o j}(\text { old })+\Delta V_{o j} .
\end{aligned}
$$

Step 10: Test the stopping condition.

For each updating of weights different training data of apparent resistivity have been generated and it has been considered as the synthetic data needed for the ANN network to train (Sivanandam et al. 2007).

The stopping condition may be the minimization of the errors, number of epochs, etc.

Here in our program initial parameters are fixed as follows.

net.trainparam $\cdot$ show $=2$;

net.trainparam.lr $=0.01$;

net.trainparam. $\mathrm{mc}=0.5$;

net.trainparam.epochs $=100$;

net.trainparam.goal $=1 \mathrm{e}-6$.

The initial set is not chosen randomly. For a given number of neurons on the hidden layer, we drive several runs and we compute the mean final criterion $J$ value, the sample standard deviation, the best (minimal) final criterion value and the energy $E$ of the network with this best final criterion value. Our FNN is defined by:

$$
\text { net }=\text { newff }(P, T, \text { Neurons }) \text {. }
$$

\subsection{Selection of the neural network}

FFBPN is a systematic method for training multilayer artificial neural networks. A feed forward neural network is a non-parametric estimation of statistical models for extracting nonlinear relations of the input data, whereas the back propagation algorithm computes the error in backward direction. Thus, the two phases of this algorithm is described below.

- Forward phase: During this phase, the free parameters of the network are fixed, and the input signal is propagated through the network. It finishes with the computation of an error signal.

$$
e_{i}=d_{i}-y_{i}
$$

where $d_{i}$ is the desired response and $y_{i}$ is the actual output produced by the network in response to the input $x_{i}$.
- Backward phase: During this second phase, the error signal $e_{i}$ is propagated through the network in the backward direction, hence the name of the algorithm. It is during this phase that adjustments are applied to the free parameters of the network to minimize the error $e_{i}$ in a statistical sense. The back propagation learning algorithm is simple to implement and computationally efficient, as its complexity is linear in assigning the synaptic weights of the network. The network is finally tuned using the entire set of training examples and then the testing is applied (Rumelhart et al. 1986; Haykin 2009).

In the present study, we have adopted FFBPN for the interpretation of the subsurface layer model and the full description of the program is shown in figure 5. In the case of generalised approach, care should be taken in fixing the input parameters, since it controls the output.

\subsection{Choice of input parameters}

The Feed-forward layer network is constructed here in our approach to analyze the observed apparent resistivity field data and while analyzing it makes changes to smooth the data by updating weights and biases of subsequent ANN network layers. Network training function trainlm (LevenbergMarquardt optimization command used in MATLAB R2008b software) is used to train the network. The specified transfer function affects the changes in training, and the optimization of the observed data is then adapted to the network and fits with the activation function of the network. We can see the performance of the network using 'perform' function. Training stops, when the minimum mean square error is achieved within a particular number of epochs.

\subsection{Training set and training samples}

The number of training samples is of direct influence on the total number of internal variables allowed in the neural networks to keep training overconstrained. The total number of internal variables should never exceed the number of training samples (Vander Baan and Jutten 2000). In the present approach, synthetic data is generated based on the field data. Figure 5 proclaims the new feature development algorithm used for generating its own synthetic data based on random variations of weights and depicting the layer model based on the slope variation parameters.

Though the earlier attempts to invert DC resistivity data using ANN were successful, the application on real data was limited, as they depend mainly on the synthetic data with less parameter variations in more specific curve types. Here we 


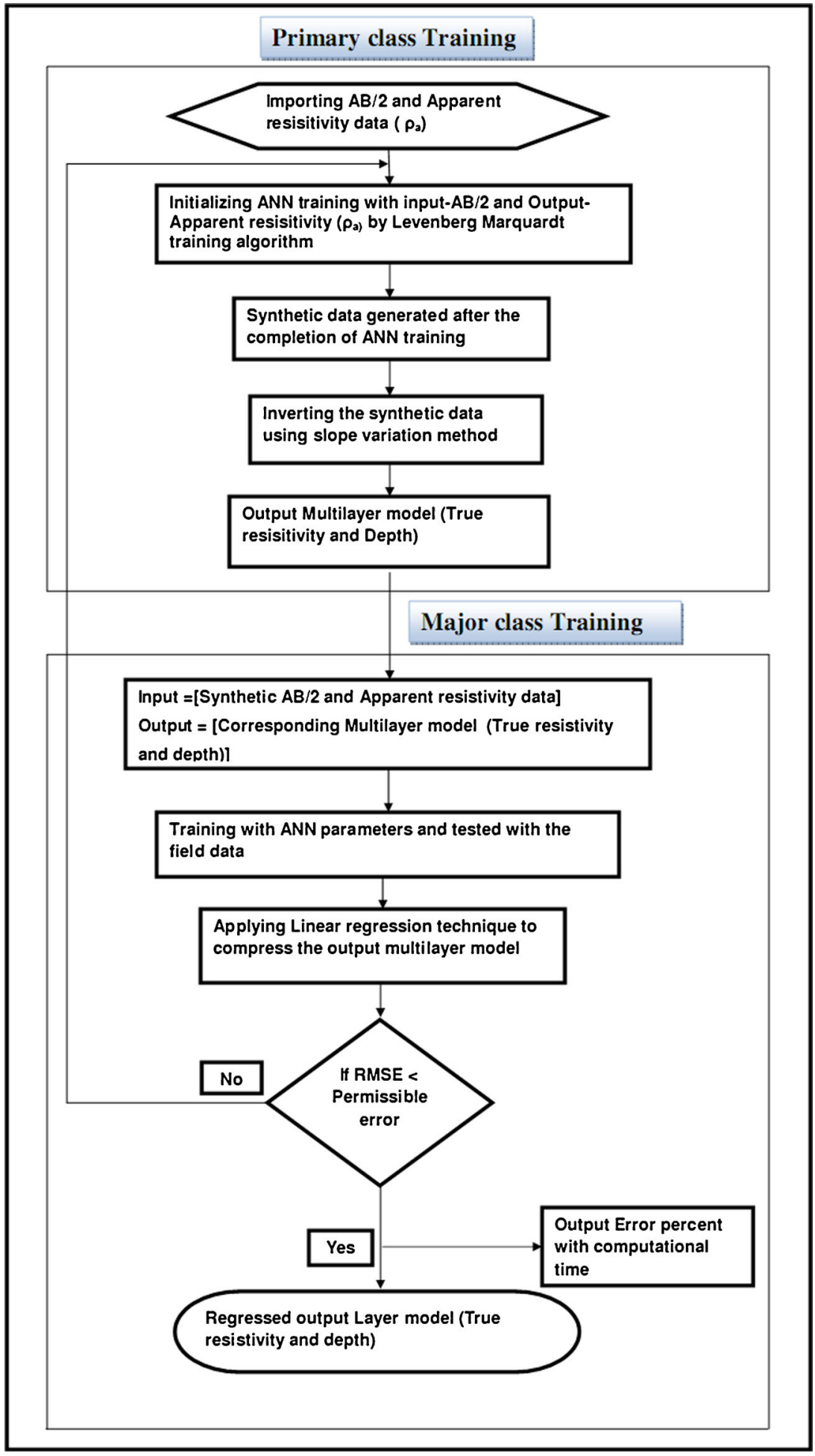

Figure 5. Artificial Neural Networks generalized algorithm for geoelectrical resistivity inversion.

made an attempt to overcome some of these limitations by designing a network that can incorporate a wide range of model parameters. In both the cases the feed-forward neural network (FFNN) is used as a mapping function between inputs and outputs. Lebvenberg-Marquardt back propagation is used as the learning rule, which is sufficient to design any kind of nonlinear classifier and is, by far, the most popular learning algorithm (Srinivas et al. 2012). Since an optimum size and efficiency of the training dataset is important to render generalization capability to the network, the database 
is generated cautiously. Adequate cross-validation has been carried out for ensuring the performance of network on new data examples.

\section{Algorithm description and application}

In the present algorithm, the training has been classified into two major parts, viz., primary and major class training.

\subsection{Primary class training}

In the primary training, the input data is processed here. In the present application of geoelectrical resistivity inversion, Vertical Electrical Sounding (VES) data (AB/2 and apparent resistivity) is fed to the primary training. Here, the raw data is smoothened by applying random weights to each input and training the input data. After the training it produces a number of synthetic data which are proportional to the number of epochs. Then a multilayer model is obtained based on the variation of slope of VES curve for each synthetic data after training as output.

Slope variation refers to the basic method for obtaining the trend of the VES curve changing with respect to the subsurface layers obtained from the field curve. Whenever the VES curve changes its trend then the slope varies and this represents the change in the subsurface layer. Initially, a multilayer model is obtained, as each and every data point obtained from the field may not be linear. The slope is normally described by the ratio of the 'rise' divided by the 'run' between two points on a line. The line may be practical - a set obtained by the $\mathrm{AB} / 2$ and apparent resistivity data values. Whenever the curve changes its behaviour, the slope changes (figure 6).

The change can be depicted as $\left(y_{2}-y_{1}\right)=\Delta y$. Similarly, for the $\mathrm{x}$ axis it is $\left(x_{2}-x_{1}\right)=\Delta x$.

Thus slope $m$ of the line is

$$
m=\frac{y_{2}-y_{1}}{x_{2}-x_{1}} .
$$

The concept of slope applies directly to grades or gradients in geophysics, geography and civil engineering. Through trigonometry, the grade $m$ of a curve is related to its angle of incline $\theta$ by

$$
m=\tan \theta \text {. }
$$

Whenever the angle of inclination $\theta$ representing the tangent deviates from 1 , nonlinearity occurs and the corresponding layer parameter is obtained $\left(\tan 45^{\circ}=1\right)$. Thus at the end of the primary training, we have more synthetic datasets with corresponding multilayer models.

\subsection{Major class training}

In the major class training, the synthetic data obtained from the primary training session is fed as an input to the FFBP networks with LevenbergMarquardt training algorithm, where the output for the FFBP algorithm is the multilayer model for the corresponding synthetic data. Moreover, other parameter limitations follow the same rule as that of parameters used in the previous session.

In the testing phase, the trained datasets were tested with the original field data and the output model is linearly regressed with each multilayer and provide a compressed layer model as an output. The specially designed algorithms provide a separate model for each iteration and the user can choose a certain model.

\subsection{Step by step procedure}

- While running the program the user can fix the number of epochs and permissible error. Number of layers is optional; when it is not mentioned the ANN algorithm produces its own model at each iteration automatically.

- The user can import the data in the Microsoft Excel format. $\mathrm{AB} / 2$ and apparent resistivity data can be imported.

- After completing the user interface, the program runs automatically. ANN inversion runs on the blueprint of the generalized ANN algorithm with certain controlling parameters, viz., number of epochs and permissible error percent.

- After ANN inversion, the outputs are shown in separate figure windows.

- Geoelectric section for the corresponding layer model is finally obtained.

- Running message will be shown in the command window while the program runs.

- After iterating the algorithm, the user can save the respective plots.

- The output performance plot can be obtained for each training dataset.

\subsection{Validation of the ANN algorithm with field data}

The designed ANN algorithm has been validated with different field datasets obtained from different geological formations. Two datasets were chosen and the results were compared with the earlier results/lithologs; the correlated results are given in table 1 . 


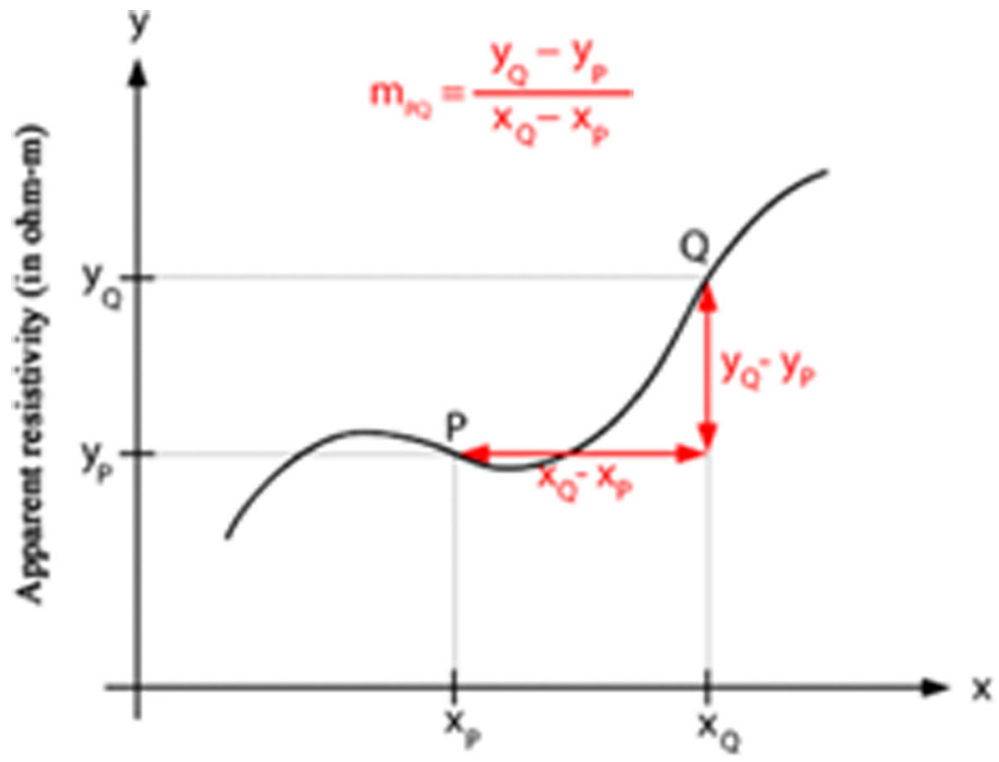

$\mathrm{AB} / 2$ (in m)

Figure 6. Slope variation model for the corresponding VES curve determination.

Table 1. ANN inversion results along with error percentage in comparison with litholog information.

\begin{tabular}{llccc}
\hline $\begin{array}{l}\text { True resistivity } \\
\text { (in } \Omega \mathrm{m})\end{array}$ & $\begin{array}{c}\text { Depth } \\
\text { (in m) }\end{array}$ & $\begin{array}{c}\text { ANN inverted error } \\
\text { percent (RMSE) }\end{array}$ & $\begin{array}{c}\text { Computational } \\
\text { time (in sec) }\end{array}$ & $\begin{array}{c}\text { Depth according } \\
\text { to litholog (in m) }\end{array}$ \\
\hline Data 1 & & & & \\
10.4029 & 3.4998 & 0.0016 & 9.5415 & 3.5 \\
1.87606 & 25 & & & \\
189.5 & & & \\
& & & \\
Data 2 & & & & \\
213.575 & 1.04413 & 5.65205 & \\
414.699 & 4.35537 & & & 36.7 \\
54.7687 & 37.5 & & \\
1459.7 & & & \\
\hline
\end{tabular}

\subsection{Performance criteria}

To achieve desired optimal neural network model Root Mean Square Error (RMSE) correlation coefficient $(R)$ and correlation of determination $\left(R^{2}\right)$ are used in the current study. They are given by:

The RMSE is the measure of the difference between values predicted by a model and the values actually observed from the environment that is being modelled. The error is calculated as the difference between the target output and the network output. The goal is to minimize the average of the sum of these errors. Here, the difference is between the ANN tested synthetic data output and the observed field data

$$
\operatorname{RMSE}=\sqrt{\sum_{i=1}^{n} \frac{(t(k)-a(k))^{2}}{n}} .
$$

Here $t(k)$ is the target output which is the observed field data, ' $n$ ' is the number of data points and $a(k)$ is the network output which is the synthetic data obtained after ANN training.

Correlation - often measured as a correlation coefficient $(r)$ - indicates the strength and direction of a linear relationship between two variables 


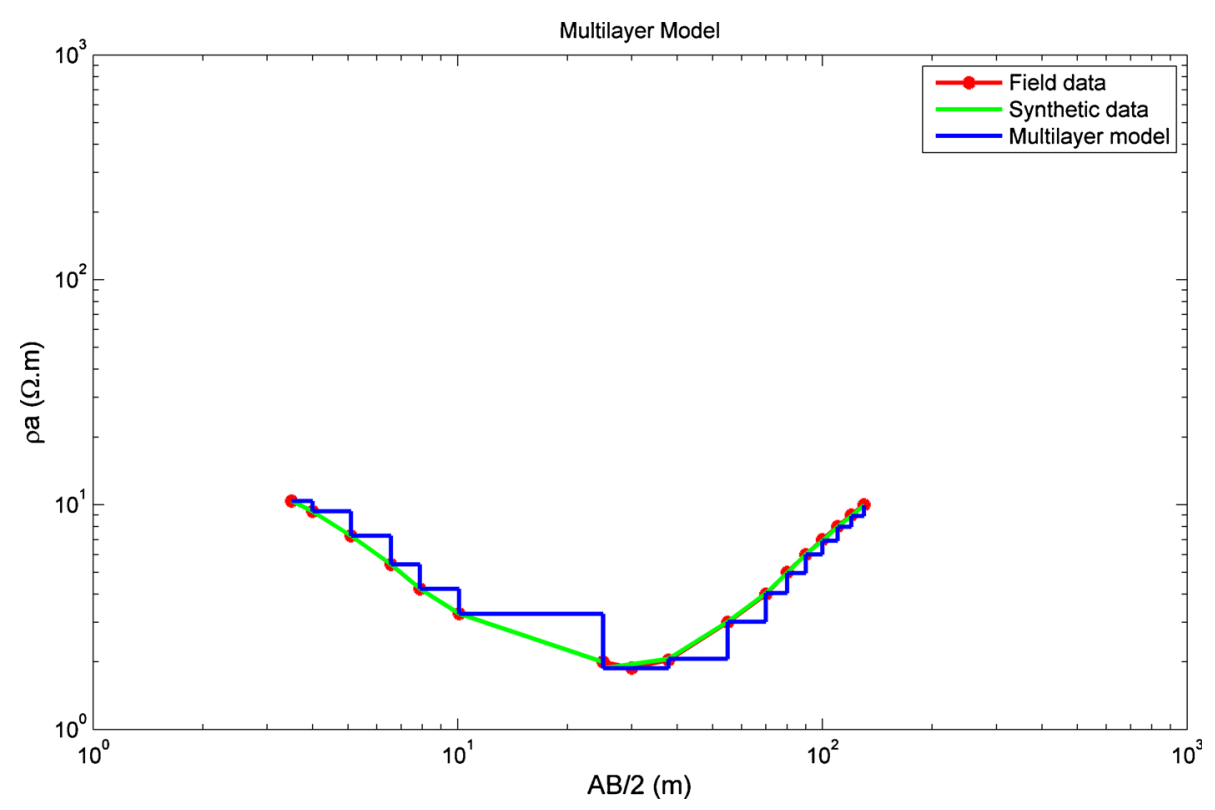

Figure 7. Multilayer model using ANN geoelectrical resistivity inversion for data 1.

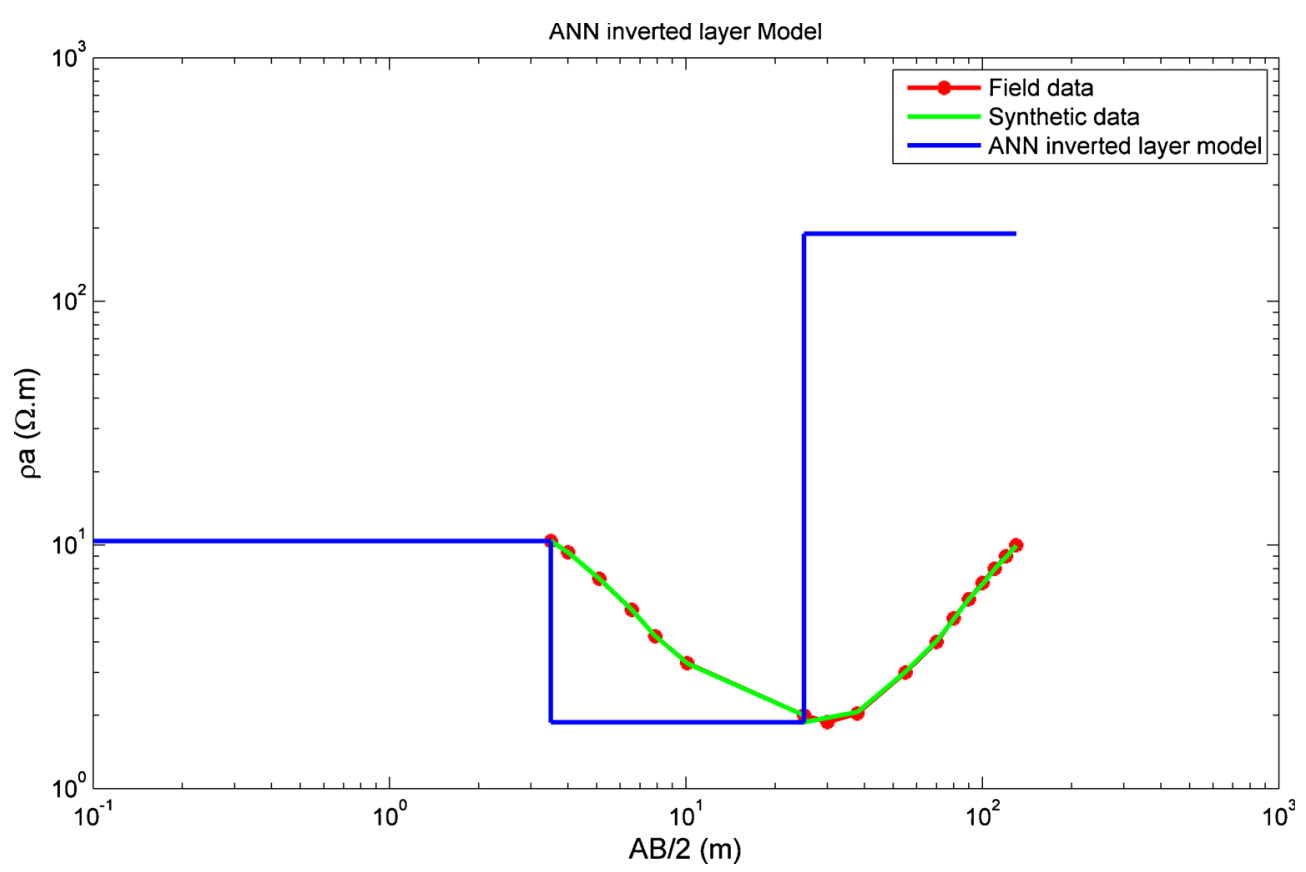

Figure 8. ANN geoelectrical resistivity inversion layer model for data 1.

(for example, model output and observed values). A number of different coefficients are used for different situations. Here, ANN trained output is correlated to targeted observed field data output.

$$
r=\frac{\sum_{i=1}^{n}\left(x_{i}-\bar{x}\right) \cdot\left(y_{i}-\bar{y}\right)}{\sqrt{\sum_{i=1}^{n}\left(x_{i}-\bar{x}\right)^{2} \cdot \sum_{i=1}^{n}\left(y_{i}-\bar{y}\right)^{2}}}
$$

where $x$ and $y$ are the target field data and the ANN trained output, respectively. $\bar{x}$ indicates $\Sigma x / n$, similarly $\bar{y}$. The correlation is +1 in the case of a perfect increasing linear relationship, and -1 in case of a decreasing linear relationship, and the values in between indicate the degree of linear relationship between, for example, model and observations. A correlation coefficient of 0 means that there is no linear relationship between the variables. The square of the correlation coefficient $\left(r^{2}\right)$, 


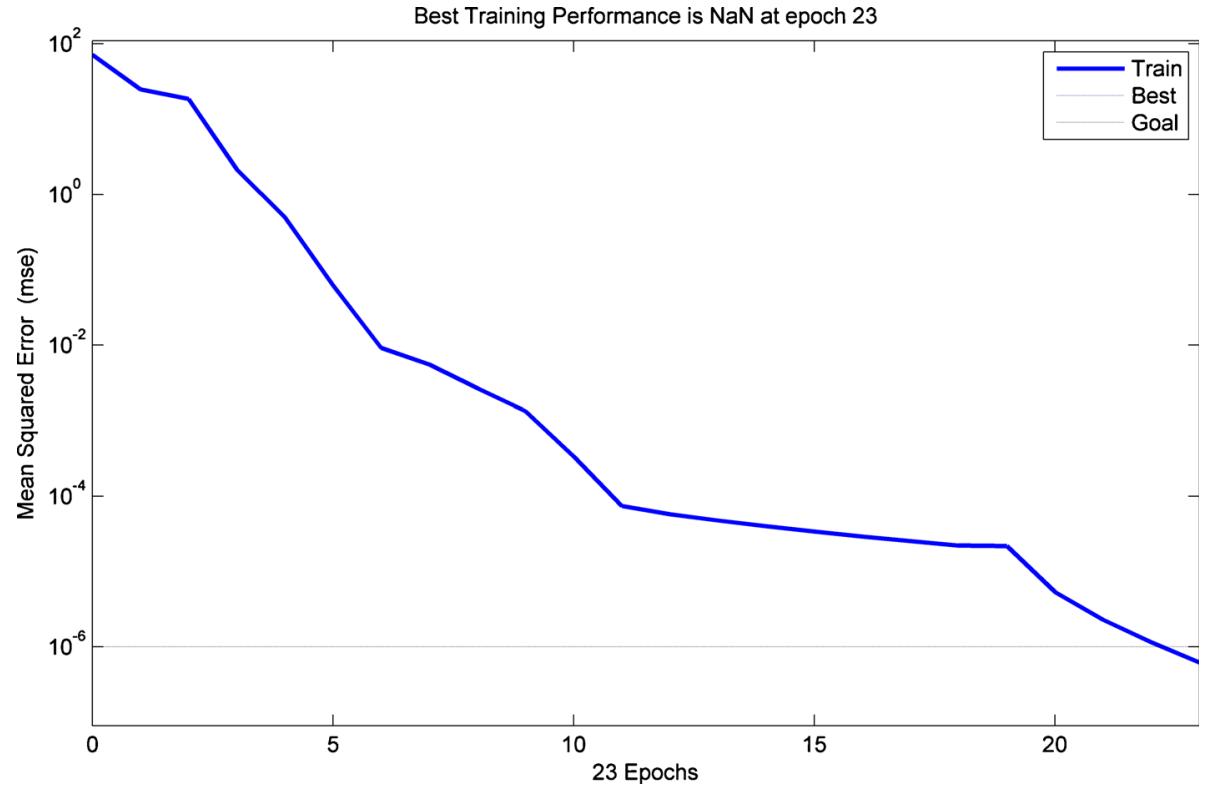

Figure 9. Performance plot of ANN geoelectric inversion for data 1.
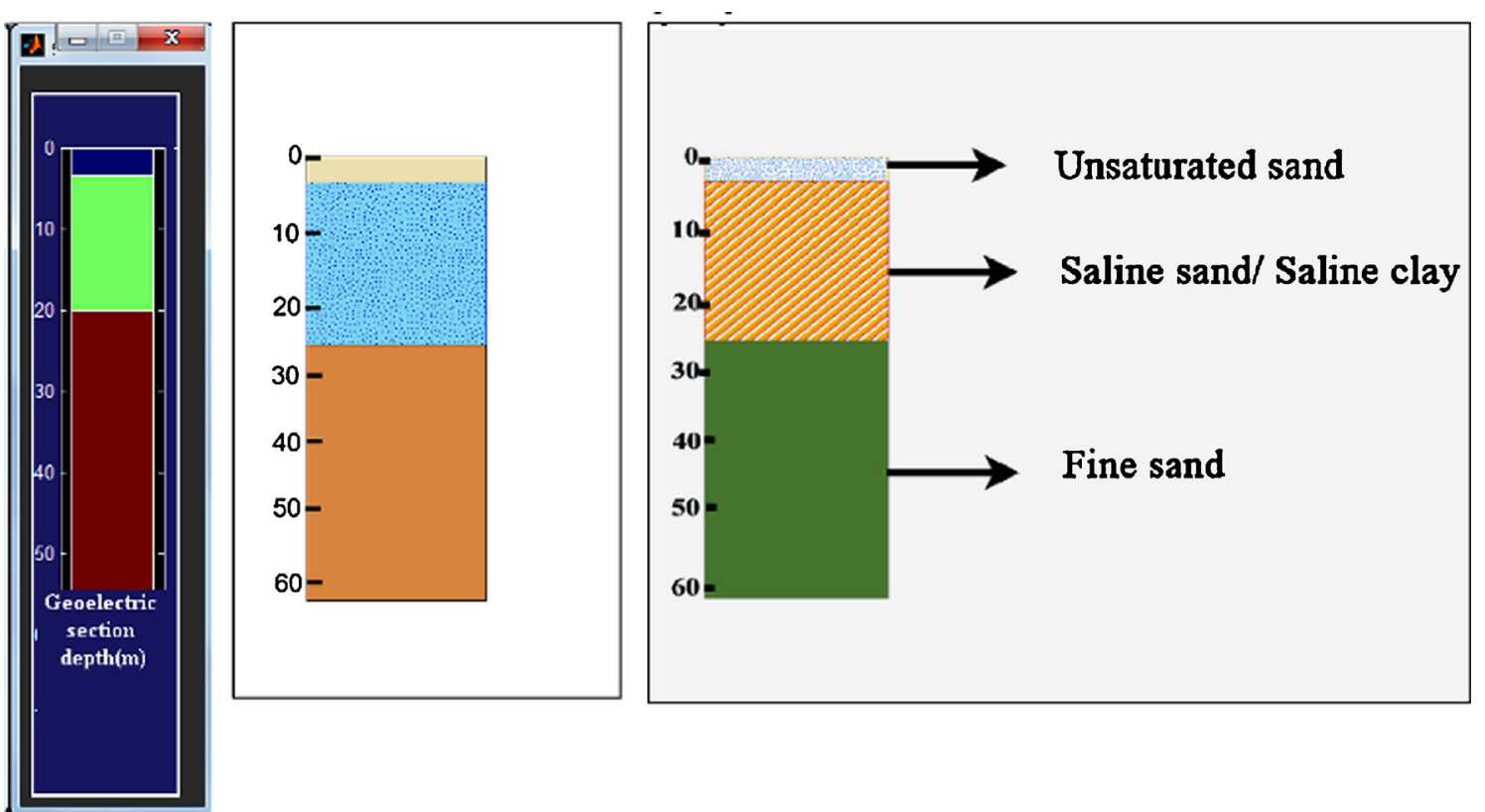

Figure 10. ANN geoelectric section output for data 1. Comparison of earlier results with litholog information (source: Kalpan Choudhury and Saha 2004).

known as the coefficient of determination, describes how much of the variance between the two variables is described by the linear fit (Vittal and Malini 2007).

\section{Results and discussions}

Linear transfer function (purelin) is used in the output layer of the ANN architecture to make the nonlinear apparent resistivity data linear by adjusting certain weights used as the parameters in the program. Network training function trainlm (Levenberg-Marquardt optimization) is used to update weight and bias values between the $\mathrm{AB} / 2$ and apparent resistivity values. Feed-forward layer network is constructed to analyze the observed field data and while analyzing it makes changes to the data by means of updating weights and biases of subsequent layers. We can see the performance of the network using the perform function. Best training performance can be achieved after 


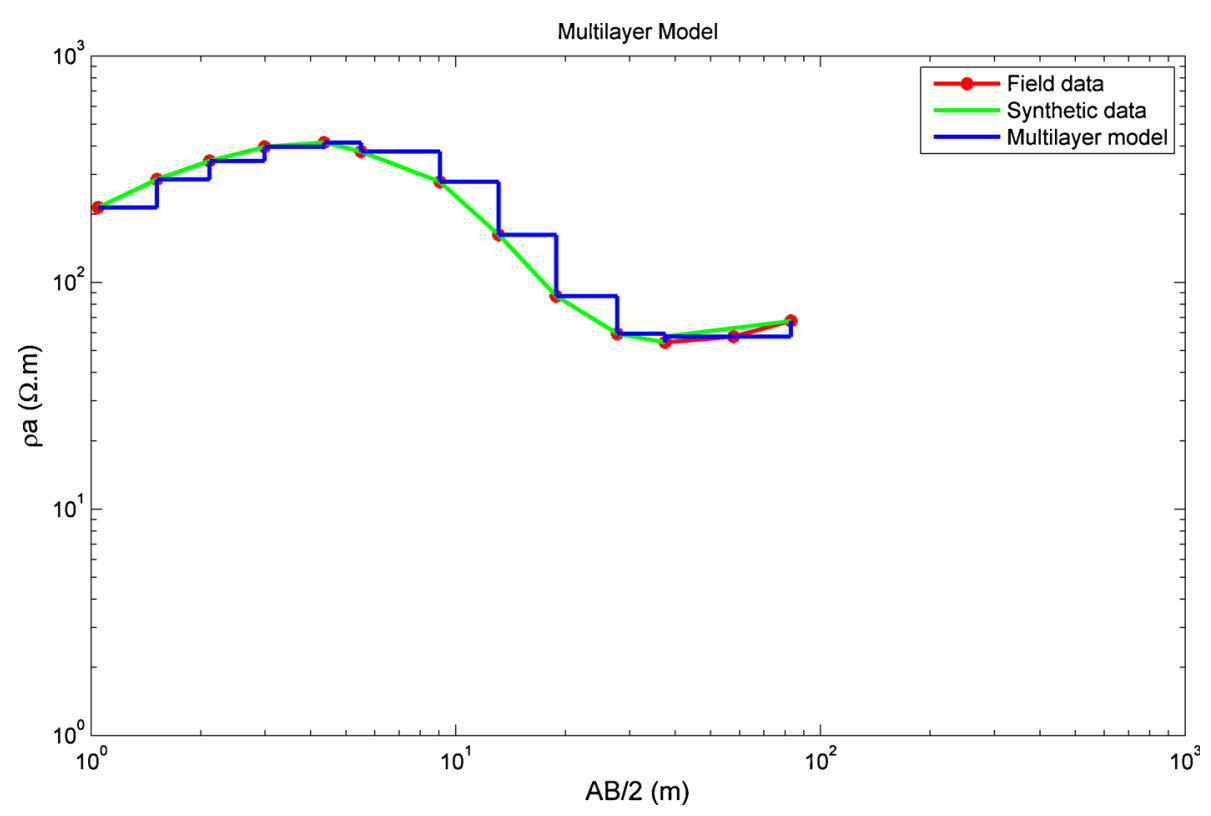

Figure 11. Multilayer model using ANN geoelectrical resistivity inversion for data 2.

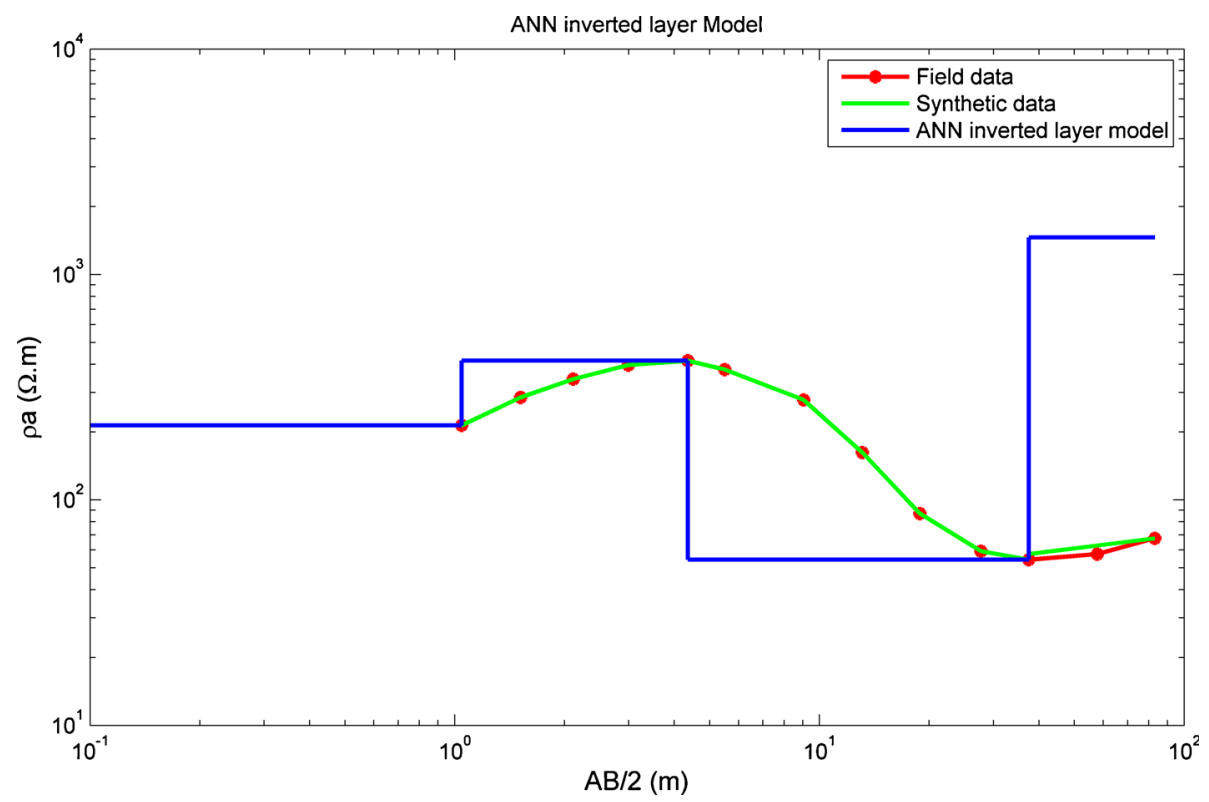

Figure 12. ANN geoelectrical resistivity inversion layer model for data 2.

the iterations have successfully completed the goal using the number of epochs for the synthetic data. The training stops whenever the goal has been achieved in a particular number of epochs. The test using trained data indicates that the ANN system can converge to the target rapidly and accurately. According to the study of $1 \mathrm{D}$ resistivity, inversion procedure using the ANN system was carried out because the procedure works well for the observed data. The synthetic and ANN trained data along with layer model was plotted and the layer model for the field data was also predicted. After testing the data, the network has to interpret the layer model of the subsurface. The well trained network will increase the performance level of the output parameters. Moreover, the network parameters weighted the output enhancement of the network.

Vertical electrical resistivity sounding data obtained from Digha-Shankarpur coastal belt of West Bengal, India (Kalpan Choudhury and Saha 2004) has been considered in the interpretation using ANN and is presented here as data 1. 


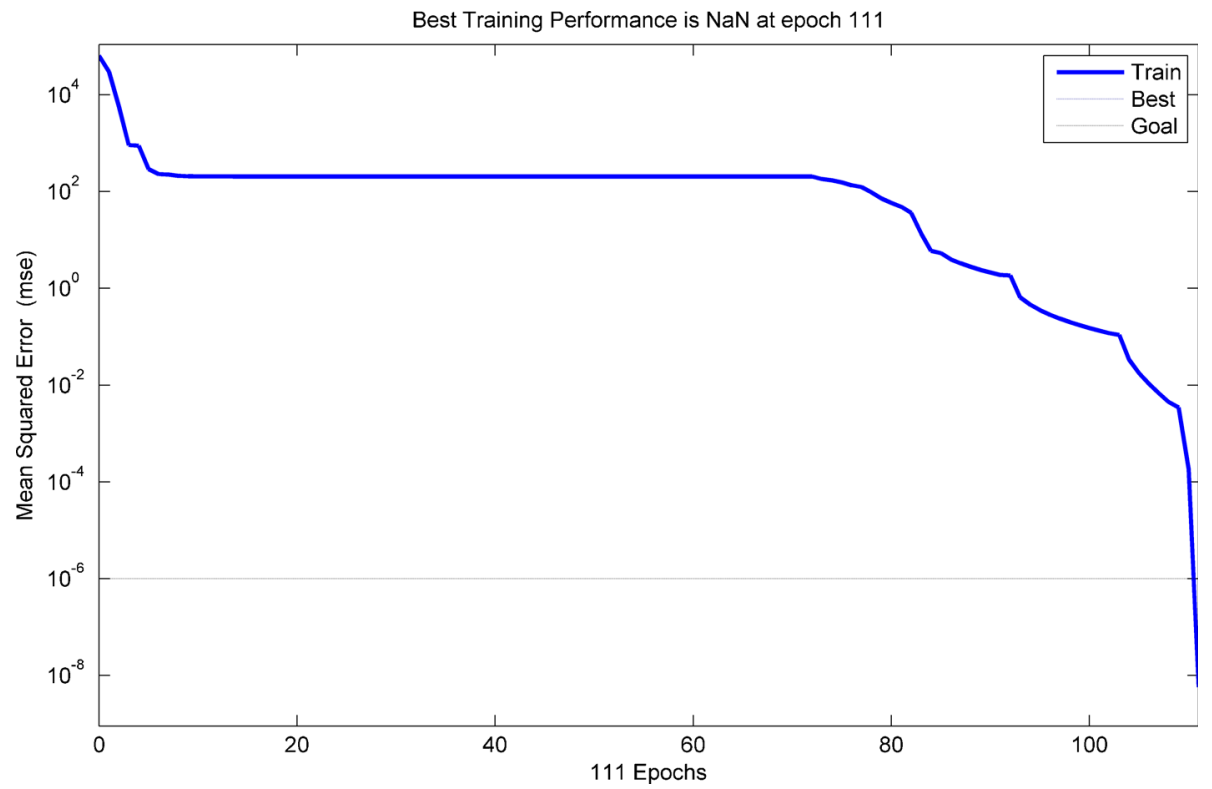

Figure 13. Performance plot of ANN geoelectric inversion for data 2.
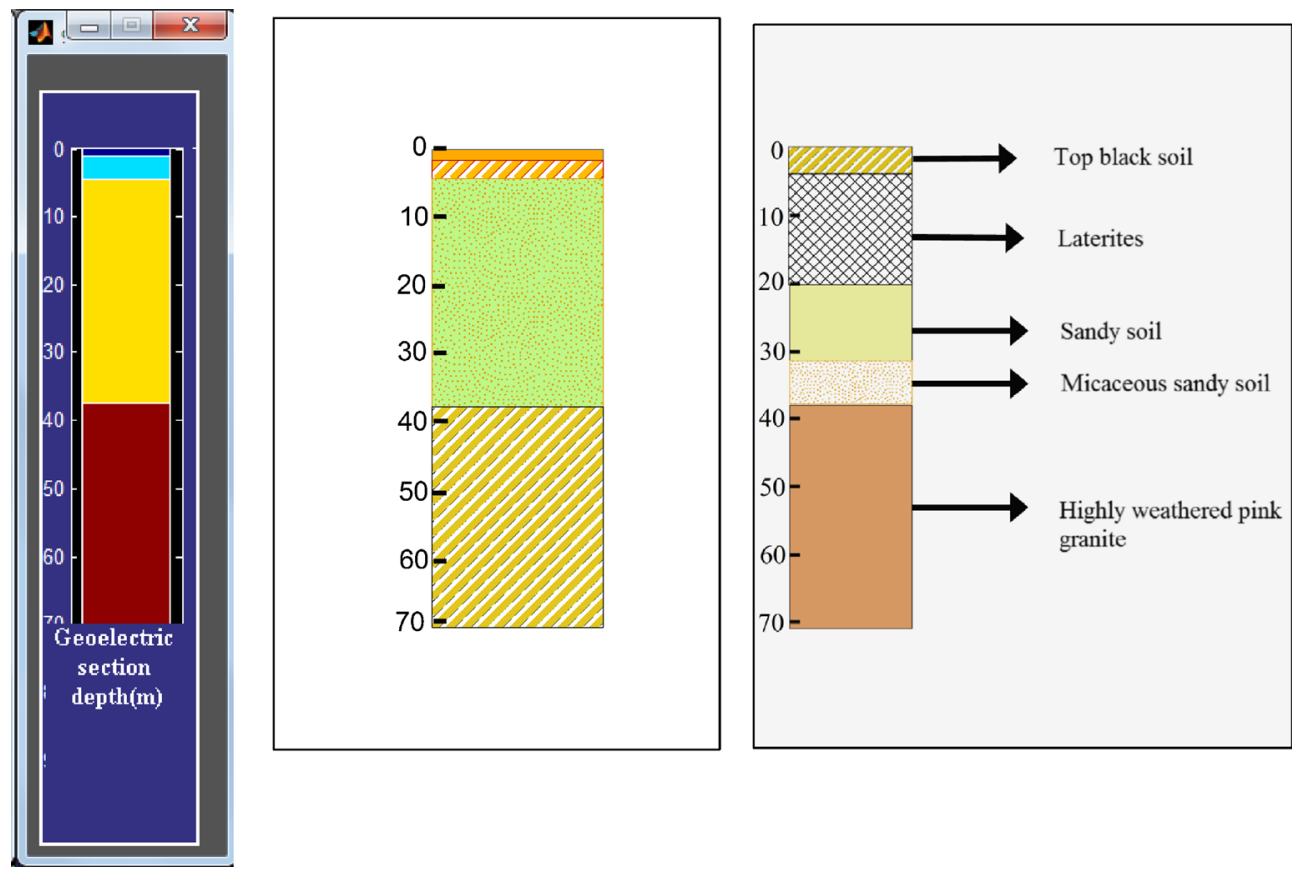

Figure 14. ANN geoelectric section output for data 2 with litholog information (source: Batte et al. 2008).

Figure 7 shows the multilayer model of the data 1 after inversion using ANN specially designed algorithm. Figure 8 shows the ANN inverted layer model along with the synthetic data generated during the training. Figure 9 shows the performance plot for interpreted data 1 . The performance represents the least square error achieved for the particular number of epochs. The error is measured in terms of RMSE. Figure 10 represents the ANN inverted geoelectric section along with litholog and earlier results.
Data 2 was obtained from Gwase/Bubeeko village of Kamuli District of Eastern Uganda (Batte et al. 2008). Figures 11 and 12 show the ANN geoelectrical inversion results of data 2 . Figure 13 shows the ANN performance plot for data 2. The correlation litholog is shown in figure 14 .

This inversion thus represents the layer parameters of subsurface geology, viz., depth and resistivity. Successful interpretation was produced only after the training and the model parameters determined with the trained data. The results of 
interpretation of the near-subsurface features by means of this ANN technique is satisfactory and is more efficient and the error has been checked with the synthetic trained data.

\section{Conclusion}

The better performance of FFBP-LM algorithm reveals the features of subsurface parameters clearly. The advantage of ANN training and testing applied here is, it can be extended to a generalized approach. For training the data itself, it depends on the updating of weights and not on the training datasets of the particular region for study. The random updating of weights thus provides enough datasets for training and the learning rate and number of epochs considered in the algorithm thus controls the training to a certain boundary. The generalized ANN algorithm presented here has been tested successfully and this powerful tool can be applied to any case study. In case study related problems, the conventional ANN is trained by the same field dataset and tested with the same field data. But this approach extends the research to a generalization. Increasing the number of epochs by the user and fixing more accuracy as the datasets generated by updating the weights completely, depends on a number of epochs. So the performance results will be more when more datasets have been trained.

\section{References}

Ahmad Neyamadpour, WAT Wan Abullah and Samsudin Taib 2010 Inversion of quasi-3D DC resistivity imaging data using artificial neural networks; J. Earth Syst. Sci. $11927-40$.

Aristodemou E, Pain C, De Oliveira C, Goddard T and Harris C 2005 Inversion of nuclear well-logging data using neural networks; Geophys. Prospect. 53 103-120.

Backus G E and Gilbert J F 1969 Uniqueness in the inversion of inaccurate gross earth data; Phil. Trans. Roy. Soc. A 266 123-192.

Batte A G, Muwanga A and Sigrist W P 2008 Evaluating the use of vertical electrical sounding as a groundwater exploration technique to improve on the certainty of borehole yield in Kamuli district (Eastern Uganda); African J. Sci. Technol. (AJST) 9 72-85.

Baum E and Hausler D 1989 What size net gives valid generalization? In: Advances in Neural Information Processing Systems I (ed.) Touretzky D, Morgan Kaufman, pp. 80-90.

Calderon-Macias C, Sen M K and Stoffa P L 2000 Artificial neural networks for parameter estimation in geophysics; Geophys. Prospect. 48 21-47.

Cranganu C 2007 Using Artificial Neural Networks to predict the presence of overpressured zones in the Anadarko Basin, Oklahoma; Pure Appl. Geophys. 164 2067-2081.
El Qady G and Ushijima K 2001 Inversion of dc resistivity data using neural networks; Geophys. Prospect. 49 417-430.

Flathe H 1955 A practical method of calculating geoelectrical model graphs for horizontally stratified media; Geophys. Prospect. 3 268-294.

Ghosh D P 1971 Inverse filter coefficients for the computation of the apparent resistivity standard curves for horizontally stratified earth; Geophys. Prospect. 19 769-775.

Haykin S 2009 Neural Networks and Learning Machines; 3rd edn, Prentice Hall.

Jimmy Stephen, Manoj C and Singh S B 2004 A direct inversion scheme for deep resistivity sounding data using artificial neural networks; Proc. Indian Acad. Sci. 113 49-66.

Kalpan Choudhury D K and Saha 2004 Integrated geophysical and chemical study of saline water intrusion; Groundwater 42 671-677.

Kosinky W K and Kelly W E 1981 Geoelectrical sounding for predicting aquifer properties; Groundwater 19 $163-171$.

Louis I F, Louis F L and Grambas A 2002 Exploring for favorable groundwater conditions in hard rock environments by resistivity imaging methods: Synthetic simulation approach and case study example; J. Electr. Electron. Eng., Spec. Issue, pp. 1-14 (http://www. geophysicsonline.gr/paper-10.pdf).

Maiti S and Tiwari R K 2008 Classification of lithofacies boundaries using the KTB borehole data: A Bayesian Neural Network Modeling; 7th International Conference and Explosition on Petroleum Geophysics, Hyderabad, 80p.

Maiti S and Tiwari R K 2009 A hybrid Monte Carlo method based artificial neural networks approach for rock boundaries identification: A case study from the KTB bore hole; Pure Appl. Geophys. 166 2059-2090.

Maiti S, Gupta G, Erram V C and Tiwari R K 2011 Inversion of Schlumberger resistivity sounding data from the critically dynamic Koyna region using hybrid Monte Carlo-based neural network approach; Nonlinear Process Geophys. 18 179-192.

Maiti S, Erram V C, Gautam Gupta, Ram Krishna Tiwari, Kulkarni U D and Sangpal R R 2012 Assessment of groundwater quality: A fusion of geochemical and geophysical information via Bayesian neural networks; Environ. Monit. Assess., doi: 10.1007/s10661-012-2802-y.

Maiti S, Gautam Gupta, Vinit C Erram and Ram Krishna Tiwari 2013 Delineation of shallow resistivity structure around Malvan, Konkan region, Maharashtra by neural network inversion using vertical electrical sounding measurements; Environ. Earth Sci. 68 779-794.

MATLAB R 2008 The Mathworks, Inc., Natick, MA.

Mazac O, Kelly W E and Landa I 1985 A hydrophysical model for relation between electrical and hydraulic properties of aquifers; J. Hydrol. 79 1-19.

Mooney H M, Orellana E, Pickett H and Tornheim L 1966 A resistivity computation method for layered earth model; Geophys. 31 192-203.

Poulton M M, Sternberg B K and Glass C E 1992 Location of subsurface targets in geophysical data using neural networks; Geophys. 57 1534-1544.

Rumelhart D E, Hinton G E and Williams R J 1986 Learning internal representation by error propagation, Parallel Distributed Processing (Cambridge, MA: MIT Press) 1 318-362.

Satyendra Narayan, Maurice B Dusseault and David C Nobes 1994 Inversion techniques applied to resistivity inverse problems; Inverse Problems 10 669-686. 
Satish Kumar 2007 Neural networks - A class room approach, Tata McGraw-Hill Publishing Ltd., New Delhi.

Sheen N 1997 Automatic interpretation of archaeological gradiometer data using a hybrid neural network, $\mathrm{PhD}$ thesis, University of Bradford.

Singh U K, Tiwari R K and Singh S B 2005 One-dimensional inversion of geo-electrical resistivity sounding data using artificial neural networks - a case study; Comput. Geosci. 31 99-108.

Singh U K, Tiwari R K and Singh S B 2010 Inversion of 2D $\mathrm{DC}$ resistivity data using rapid optimization and minimal complexity neural network; Nonlinear Process Geophys. $171-12$.

Sivanandam S N, Sumathi S and Deepa S N 2007 Introduction to Neural Networks using MATLAB 6.0; 3rd edn, Chapter 8, Feed forward Networks, Tata McGraw Hill Publishing Company Ltd.

Sreekanth P D, Geethanjali N, SreeDevi P D, Ahmed Sreekanth Shakeel, Ravikumar N and Kamala Jayanthi P D 2009 Forecasting groundwater level using artificial neural networks; Curr. Sci. 96 933-939.

Srinivas Y, Stanley Raj A, Hudson Oliver D, Muthuraj D and Chandrasekar N 2010 An application of Artificial Neural Network for the interpretation of three layer electrical resistivity data using Feed Forward Back Propagation Algorithm; Curr. Dev. Artif. Intel. 1 1-11.

Srinivas Y, Stanley Raj A, Hudson Oliver D, Muthuraj D and Chandrasekar N 2012 A robust behavior of Feed Forward Back propagation algorithm of Artificial Neural Networks in the application of vertical electrical sounding data inversion; Geosci. Frontiers 3(5) 729-736.
Sri Niwas and Singhal D C 1981 Estimation of aquifer transmissivity from Dar-Zarrouk parameters in porous media; J. Hydrol. 50 393-399.

Telford W M, Geldart L P and Sheriff R E 1990 Applied Geophysics; 2nd edn, Cambridge University Press, Cambridge.

Tikhonov A N and Arsenin V Y 1977 Solution of Ill-Posed Problems; Winston, Washington DC.

Van Dam J C 1964 A simple method for the calculation of standard graphs to be used in geoelectrical prospecting; Ph.D thesis, Delft Technological University, The Netherlands.

Vander Baan M and Jutten C 2000 Neural networks in geophysical applications; Geophys. 65 1032-1047.

Vittal P R and Malini V 2007 Statistical and numerical methods; Margham Publications, Chennai, 13.1-13. $61 \mathrm{p}$.

Weiland A and Leighton R 1987 Geometric analysis of neural network capabilities; IEEE 1st International Conference on Neural Networks.

Werbos P J 1974 Beyond regression: New tools for prediction and analysis in the behavioral sciences; PhD thesis, Harvard University.

Yadav G S and Abolfazli H 1998 Geoelectrical soundings and their relationships to hydraulic parameters in semi arid regions of Jalore, northwest India; J. Appl. Geophys. $3935-51$.

Yegnanarayana B 2005 Artificial Neural Networks; Prentice Hall of India Private Limited, New Delhi. http://www.ualberta.ca/ unsworth / UA-classes / 424 / labs424-2012.html. 